\title{
A Robust Spectral Method for Pricing of American Put Options on Zero-Coupon Bonds
}

\author{
Edson Pindza and Kailash C. Patidar* \\ Department of Mathematics and Applied Mathematics, University of the Western \\ Cape, Private Bag X17, Bellville 7535, South Africa.
}

Received 17 May 2016; Accepted (in revised version) 20 October 2017.

\begin{abstract}
American put options on a zero-coupon bond problem is reformulated as a linear complementarity problem of the option value and approximated by a nonlinear partial differential equation. The equation is solved by an exponential time differencing method combined with a barycentric Legendre interpolation and the Krylov projection algorithm. Numerical examples shows the stability and good accuracy of the method.
\end{abstract}

AMS subject classifications: 39A05, 65M06, 65M12, 91G60

Key words: Interest rate model, American put bond options, zero-coupon bond, barycentric Legendre method, Greeks.

\section{Introduction}

A bond is a financial instrument which allows an investor to loan money to an entity (a corporate or governmental) that borrows the funds for a period of time at a fixed interest rate (the coupon) and agrees to pay a fixed amount (the principal) to the investor at maturity. A zero-coupon bond is a bond that makes no periodic interest payments. A bond option is a financial contract which gives the holder the right but not the obligation to buy or sell a bond at a certain price on or before the option expiry date. A European bond option is an option to buy or sell a bond at maturity for a fixed price. On the other hand, an American bond option offers a possibility to buy or sell a bond for a predetermined price on or before the maturity date. A buyer of a bond call option is expecting a decline in interest rates and an increase in bond prices, while a buyer of a put bond option is expecting an increase in interest rates and a decrease in bond prices.

Pricing interest rate contingent claims is a popular field of research in finance [20]. For European zero-coupon bonds the prices of these claims can be calculated as expected terminal payoffs discounted by using the path of the instantaneous risk-free rate $r$ under a risk-adjusted or equivalent martingale measure $[12,25]$. American options can be exercised at any time prior to the expiration date, which creates uncertainty and leads to

${ }^{*}$ Corresponding author. Email address: kpatidar@uwc.ac.za (K. C. Patidar) 
substantial difficulties in analytical description of this problem. Nevertheless, the information required can be obtained from an optimal exercise curve related to a free boundary problem. Therefore, numerical or semi-analytical approximation methods have to be involved in the pricing of American style contracts. The valuation of bonds with embedded options have been studied by projective successive over relaxed (PSOR) methods [28], the lattice method [4], explicit finite difference methods [6]. However, in spite of easy implementation, these methods have a slow convergence rate.

In this paper, a barycentric Legendre spectral collocation method is used for numerical valuation of American bond pricing options. Penalising the partial differential complementary problem of American bond options similarly to Ref. [2], we obtain a nonlinear partial differential equation (PDE) and derive its approximate solution in a fixed domain by the collocation scheme mentioned. To discretise the PDE in time, we employ the exponential time differencing method (ETD) studied by Cox and Matthews [8]. A modification of this scheme has been proposed by Kassman and Trefethen [13]. The class of explicit multistep exponential and explicit exponential Runge-Kutta methods have been discussed by Hochbruck and Ostermann [11]. Tangman et al. [22] used ETD methods to study European barrier and butterfly spread options for the Black Scholes model and Merton's jumpdiffusion model. American style barrier options have been discussed by Gondal [9] and Rambeerich et al. [17], who compared the exponential integrators with traditional CrankNicolson methods. Here we construct a Krylov subspace by the Arnoldi shift-and-invert method [5] and use it in the evaluation of exponential integrators.

This paper is organized as follows. In Section 2, mathematical models of zero-coupon bonds are presented and American put options on a bond for a short rate model is described. Section 3 deals with a spectral collocation method for these models. In Section 4, we discretise the corresponding systems by exponential time integrators. Numerical results are reported in Section 5, and concluding remarks are in Section 6.

\section{Pricing Models of a Zero-Coupon Bond and Options}

Let us consider the pricing of zero-coupon bonds and European bond options in the case where a short term risk-free interest rate $r$ is described by the generalized Chan-KarolyiLongstaff-Schwartz model

$$
d r(t)=\kappa(\theta-r(t)) d t+\sigma r(t)^{\gamma} d W(t)
$$

where $W(t)$ is a Wiener process, $\kappa$ the mean-reversion speed, $\theta$ the long-term interest rate, $\sigma$ the volatility, and $\gamma$ a parameter used for the nesting of term structure models within the framework of the stochastic differential equation (2.1). The pricing equations for zero-coupon bonds and European options on the bonds can be established by traditional no-arbitrage arguments. The pricing equation for both financial products is the same but the boundary conditions differ $-\mathrm{cf}$. [18]. If $\tau^{*}=T^{*}-t \in\left[0, T^{*}\right]$ denotes the time of the bond expiration, then the partial differential equation of the bond price $B\left(r, \tau^{*}\right)$ with the 
initial face value $E$ is

$$
\frac{\partial B}{\partial \tau^{*}}=\frac{1}{2} \sigma^{2} r^{2 \gamma} \frac{\partial^{2} B}{\partial r^{2}}+\kappa(\theta-r) \frac{\partial B}{\partial r}-r B, \quad B(r, 0)=E .
$$

If $\gamma=0$, the Vasicek model [24] and the analytic expression of zero-coupon bond price is

$$
B\left(r, \tau^{*}\right)=A\left(\tau^{*}\right) e^{-D\left(\tau^{*}\right) r},
$$

where

$$
A\left(\tau^{*}\right)=E \exp \left(-\theta\left[\tau^{*}-D\left(\tau^{*}\right)\right]-\frac{\sigma^{2}}{4 \kappa}\left[D^{2}\left(\tau^{*}\right)-\frac{2}{\kappa}\left(\tau^{*}-D\left(\tau^{*}\right)\right)\right]\right)
$$

and

$$
D\left(\tau^{*}\right)=-\frac{1}{\kappa}\left(e^{-\kappa \tau^{*}}-1\right) .
$$

If $\gamma=1 / 2$, the equation (2.1) represents the Cox, Ingersoll and Ross (CIR) model [7] and the straight bond value is

$$
B\left(r, \tau^{*}\right)=A\left(\tau^{*}\right) e^{-D\left(\tau^{*}\right) r},
$$

where

$$
A\left(\tau^{*}\right)=E\left[\frac{\phi_{1} e^{\phi_{2} \tau^{*}}}{\phi_{2}\left(e^{\phi_{1} \tau^{*}}-1\right)+\phi_{1}}\right]^{\phi_{3}}, \quad D\left(\tau^{*}\right)=\frac{e^{\phi_{1} \tau^{*}}-1}{\phi_{2}\left(e^{\phi_{1} \tau^{*}}-1\right)+\phi_{1}},
$$

with

$$
\phi_{1}=\sqrt{\kappa^{2}+2 \sigma^{2}}, \quad \phi_{2}=\left(\kappa+\phi_{1}\right) / 2, \quad \phi_{3}=2 \bar{\theta} / \sigma^{2}, \quad \bar{\theta}=\kappa \theta .
$$

If $\tau=T-t \in[0, T]\left(T<T^{*}\right)$ denotes the time of expiry of a bond option, the European put bond option $V(r, \tau)$ with maturity $T$ and exercise $K$ satisfies the pricing equation

$$
\begin{aligned}
& \frac{\partial V}{\partial \tau}=\frac{1}{2} \sigma^{2} r^{2 \gamma} \frac{\partial^{2} V}{\partial r^{2}}+\kappa(\theta-r) \frac{\partial V}{\partial r}-r V, \\
& V(r, 0)=\max \left\{K-B\left(r, T^{*}-T\right), 0\right\} .
\end{aligned}
$$

Let us now consider American put option $V(r, \tau)$ on a zero-coupon bond with strike price $K$, where the holder can receive a fixed payoff $g(r, \tau)$ before the expiry date $T$. Denoting by $\mathscr{L}$ the degenerate parabolic PDE operator

$$
\mathscr{L} V:=\frac{\partial V}{\partial \tau}-\left[\frac{1}{2} \sigma^{2} r^{2 \gamma} \frac{\partial^{2} V}{\partial r^{2}}+\kappa(\theta-r) \frac{\partial V}{\partial r}-r V\right],
$$

one can reformulate the option pricing problem as a differential linear complementary problem

$$
\begin{aligned}
& \mathscr{L} V(r, \tau) \geq 0, \quad V(r, \tau)-\mathrm{g}(r, \tau) \geq 0, \\
& \mathscr{L} V(r, \tau) \cdot(V(r, \tau)-\mathrm{g}(r, \tau))=0, \\
& V(r, 0)=g(r, 0)=\max \left\{K-B\left(r, T^{*}-T\right), 0\right\} \\
& V(r, \tau)=g(r, \tau), \quad r \rightarrow 0 \\
& V(r, \tau)=g(r, \tau), \quad r \rightarrow \infty
\end{aligned}
$$


where $g(r, \tau)=\max \left\{K-B\left(r, T^{*}-(T-\tau)\right), 0\right\}-\mathrm{cf}$. Ref. [28]. Further, let us add a smooth penalty term suggested by Kovalov et. al [14] and consider the arising nonlinear problem on a bounded fixed domain, so that

$$
\begin{aligned}
& \mathscr{L} V_{\epsilon}-\left(\frac{1}{\epsilon}\left[g-V_{\epsilon}\right]^{+}\right)^{p}=0,0<r<R ; 0 \leq t \leq T, \\
& V_{\epsilon}(r, 0)=\max \left\{K-B\left(r, T^{*}-T\right), 0\right\} ; 0<r<R, \\
& V_{\epsilon}(0, \tau)=g(0, \tau) ; 0 \leqslant \tau \leqslant T, \\
& V_{\epsilon}(R, \tau)=g(R, \tau) ; 0 \leqslant \tau \leqslant T,
\end{aligned}
$$

where $0<\epsilon \ll 1$ is the penalty constant, $\left[g-V_{\epsilon}\right]^{+}=\max \left\{g-V_{\epsilon}, 0\right\}, p \geq 1$ the power penalty term and $R$ a sufficiently large number - cf. Ref. [28].

\section{Barycentric Legendre Spectral Collocation Method}

In this section, we present a fast and efficient interpolation algorithm based on Legendre-Gauss-Lobatto points.

\subsection{A modified Lagrange formula}

The classical Lagrange polynomials [15] interpolating a function $u(x)$ at distinct points $x_{j}, j=0,1, \cdots, N$ are defined by

$$
p_{N}(x)=\sum_{j=0}^{N} u_{j} \ell_{j}(x), \quad \ell_{j}(x)=\prod_{\substack{k=0 \\ k \neq j}}^{N} \frac{x-x_{k}}{x_{j}-x_{k}},
$$

such that $\ell_{j}\left(x_{k}\right)=\delta_{j k}, j, k=0,1, \cdots N$, where $\delta_{j k}$ is the Kronecker delta. Although very powerful, this approach requires $\mathscr{O}\left(N^{2}\right)$ operations of addition and multiplication for each evaluation of $p_{N}(x)$, and the whole process has to be performed over again if the data $\left(x_{j}, f_{j}\right)$ are changed. Therefore, a few modifications of the interpolation procedure have been proposed - cf. Refs. [10,26]. In what follows, we use the barycentric interpolation developed in Ref. [3]. This approach requires only $\mathscr{O}(N)$ operations for the evaluation of $p_{N}(x)$. Following [3], we set $\ell(x):=\prod_{j=0}^{N}\left(x-x_{j}\right)$ and define the barycentric weights $\lambda_{j}$, $j=0,1, \cdots, N$ by $\lambda_{j}=1 / \prod_{\substack{k=0 \\ k \neq j}}^{N}\left(x_{j}-x_{k}\right)$. Then $\ell_{j}(x)=\lambda_{j} \ell(x) /\left(x-x_{j}\right)$ and the Lagrange formula (3.1) takes the form

$$
p_{N}(x)=\ell(x) \sum_{j=0}^{N} \frac{\lambda_{j}}{x-x_{j}} u_{j} .
$$

Applying it to the function $u(x) \equiv 1$, one obtains

$$
1=\sum_{j=0}^{N} \ell_{j}(x)=\ell(x) \sum_{j=0}^{N} \frac{\lambda_{j}}{x-x_{j}},
$$


so that

$$
p_{N}(x)=\left(\sum_{j=0}^{N} \frac{\lambda_{j}}{x-x_{j}} u_{j}\right) /\left(\sum_{j=0}^{N} \frac{\lambda_{j}}{x-x_{j}}\right) .
$$

This barycentric form of the Lagrange formula is frequently used in practical computations and for certain particular sets of points the barycentric weights $\lambda_{j}$ can be computed analytically. For example, let $\left\{x_{j}\right\}_{j=0}^{N}$ be the Legendre-Gauss-Lobatto points - i.e. the zeros of the polynomial $\left(1-x^{2}\right) P_{N}^{\prime}(x)$, where $P_{N}(x)$ is the Legendre polynomial of the degree $N$.

Lemma 3.1 (cf. Wang \& Huybrechs [27]). If $\left\{x_{j}\right\}_{j=0}^{N}$ are the Legendre-Gauss-Lobatto points, then

$$
\lambda_{j}=\frac{(-1)^{j} \sqrt{2}}{\sqrt{N(N+1)}} \frac{1}{P_{N}\left(x_{j}\right)}, \quad j=0,1, \cdots, N .
$$

\subsection{Differentiation matrices}

An approximate solution $u$ of the semi-discrete version of PDE (2.5) is sought in the form

$$
u(x)=\sum_{j=0}^{N} u_{j} \ell_{j}(x)
$$

so that the derivatives of $u$ are

$$
u^{\prime}(x)=\sum_{j=0}^{N} u_{j} \ell_{j}^{\prime}(x), \quad u^{\prime \prime}(x)=\sum_{j=0}^{N} u_{j} \ell_{j}^{\prime \prime}(x) .
$$

The barycentric formula for $\ell_{j}$,

$$
\ell_{j}(x)=\left(\frac{\lambda_{j}}{x-x_{j}}\right) /\left(\sum_{k=0}^{N} \frac{\lambda_{k}}{x-x_{k}}\right),
$$

yields

$$
\ell_{j}(x) \sum_{k=0}^{N} \lambda_{k} \frac{x-x_{i}}{x-x_{k}}=\lambda_{j} \frac{x-x_{i}}{x-x_{j}},
$$

and if we denote by $s$ the sum in the left-hand side of (3.3), then

$$
\begin{aligned}
& \ell_{j}^{\prime}(x) s(x)+\ell_{j}(x) s^{\prime}(x)=\lambda_{j}\left(\frac{x-x_{i}}{x-x_{j}}\right)^{\prime} \\
& \ell_{j}^{\prime \prime}(x) s(x)+2 \ell_{j}^{\prime}(x) s^{\prime}(x)+\ell_{j}(x) s^{\prime \prime}(x)=\lambda_{j}\left(\frac{x-x_{i}}{x-x_{j}}\right)^{\prime \prime} .
\end{aligned}
$$


To find the entries of the differentiation matrices, we evaluate the expressions in (3.4) at the points $x=x_{i}$, obtaining systems of linear equations. The solutions of these systems are

$$
s\left(x_{i}\right)=\lambda_{i}, \quad s^{\prime}\left(x_{i}\right)=\sum_{\substack{k=0 \\ k \neq i}}^{N} \lambda_{k} /\left(x_{i}-x_{k}\right), \quad s^{\prime \prime}\left(x_{i}\right)=-2 \sum_{\substack{k=0 \\ k \neq i}}^{N} \lambda_{k} /\left(x_{i}-x_{k}\right)^{2},
$$

or

$$
\ell_{j}\left(x_{i}\right)=0, \quad \ell_{j}^{\prime}\left(x_{i}\right)=\frac{\lambda_{j} / \lambda_{i}}{x_{i}-x_{j}}, \quad \ell_{j}^{\prime \prime}\left(x_{i}\right)=-2 \frac{\lambda_{j} / \lambda_{i}}{x_{i}-x_{j}}\left(\sum_{\substack{k=0 \\ k \neq i}}^{N} \frac{\lambda_{k} / \lambda_{i}}{x_{i}-x_{k}}-\frac{1}{x_{i}-x_{j}}\right),
$$

if $i \neq j$ and

$$
\ell_{j}\left(x_{i}\right)=1, \quad \ell_{j}^{\prime}\left(x_{j}\right)=-\sum_{i \neq j}^{N} \ell_{j}^{\prime}\left(x_{i}\right), \quad \ell_{j}^{\prime \prime}\left(x_{j}\right)=-\sum_{i \neq j}^{N} \ell_{j}^{\prime \prime}\left(x_{i}\right),
$$

if $i=j$. Thus the entries of the first and second order differentiation matrices $D^{(1)}$ and $D^{(2)}$ have the form

$$
D_{i j}^{(1)}=\ell_{j}^{\prime}\left(x_{i}\right), \quad D_{i j}^{(2)}=\ell_{j}^{\prime \prime}\left(x_{i}\right) .
$$

Let us now recall that the Legendre-Gauss-Lobatto collocation points $y_{k}, k=0,1, \cdots, N$ are clustered at the ends of the interval $[-1,1]$. On the other hand, a better approximation can be obtained, if the collocation points are located in the region of rapid change. In order to relocate the collocation points to a new region, we will use the conformal map $g$,

$$
x=g(y)=\beta+\frac{1}{\alpha} \sinh (\bar{\lambda}(y-\mu)),
$$

where $\bar{\lambda}=(\gamma+\delta) / 2, \mu=(\gamma-\delta) /(\gamma+\delta), \gamma=\sinh ^{-1}(\alpha(1+\beta)), \delta=\sinh ^{-1}(\alpha(1-\beta))$, and $\alpha$ and $\beta$, respectively, determine the location and magnitude of the region of rapid change — cf. Ref. [23].

\subsection{American interest rate options on a zero-coupon bond}

Let us now discretise our problem in the interest rate space by the barycentric Legendre spectral collocation method. We consider any real numbers $R_{m}$ and $R_{M}, R_{M}>R_{m}$, transfer the points $x_{k}=g\left(y_{k}\right) \in[-1,1], k=0,1, \cdots, N$ into interval $\left[R_{m}, R_{M}\right]$ by

$$
x_{k} \rightarrow r\left(x_{k}\right)=\frac{x_{k}+1}{2} R_{M}+\frac{1-x_{k}}{2} R_{m},
$$

and compute the first and second differentiation matrices

$$
D_{r}^{(1)}=\left(\frac{2}{R_{M}-R_{m}}\right) D_{x}^{(1)}, \quad D_{r}^{(2)}=\left(\frac{2}{R_{M}-R_{m}}\right)^{2} D_{x}^{(2)} .
$$


To simplify the notation, we write $D^{(1)}$ for $D_{r}^{(1)}, D^{(2)}$ for $D_{r}^{(2)}$ and introduce the matrix operators

$$
\begin{aligned}
& \mathbf{V}=\left[V_{1}, V_{2} \cdots, V_{N-1}\right]^{T}, \\
& D^{(1)}=\left(D_{i j}^{(1)}\right) ; j, k=1, \cdots, N-1, \\
& D^{(2)}=\left(D_{i j}^{(2)}\right) ; j, k=1, \cdots, N-1, \\
& P=\frac{1}{2} \sigma^{2} \operatorname{diag}\left\{r_{j}^{2 \gamma}\right\}, Q=\operatorname{diag}\left\{\kappa\left(\theta-r_{j}\right)\right\} ; j=1, \cdots, N-1 .
\end{aligned}
$$

Now we approximate the problem (2.5) as follows

$$
\begin{aligned}
& \dot{\mathbf{V}}=P D^{(2)} \mathbf{V}+Q D^{(1)} \mathbf{V}-r I \mathbf{V}+\left(\frac{1}{\epsilon}[\mathbf{g}-\mathbf{V}]^{+}\right)^{p}, \\
& e V^{0}=\max \left\{K-B\left(r, T^{*}-T\right), 0\right\}, \quad 0<r<R, \quad 1 \leq j \leq N-1, \\
& V_{0}=g(0, \tau), \quad V_{N}=g(R, \tau),
\end{aligned}
$$

and write it as

$$
\dot{u}=\mathbf{L} u+\mathbf{N}(u, t),
$$

where $\mathbf{L} u$ and $\mathbf{N}(u, t)$ are, respectively, linear and nonlinear parts of the problem (3.7) with the boundary conditions included. Note that the application of explicit time integrators to system (3.8) is not efficient because of the stiffness issues while the corresponding mesh is refined in the space direction. Therefore, for the time integration, an exponential time differencing (ETD) method is used.

\section{An Exponential Time Differencing Method}

The exponential time differencing methods are based on the construction of multistep integrators by using the method of variation of parameters. In this section, we follow the scheme presented in Ref. [16] but use Lagrange interpolation polynomials instead of Newton polynomials. If $h>0$, the exact solution of the problem (3.8) in the interval $\left[t_{n}, t_{n+1}\right]=\left[t_{n}, t_{n}+h\right]$ is

$$
\begin{aligned}
u\left(t_{n+1}\right) & =e^{\mathbf{L} h} u\left(t_{n}\right)+e^{t_{n} \mathbf{L}} \int_{t_{n}}^{t_{n}+h} e^{-\tau \mathbf{L}} \mathbf{N}(\tau, u(\tau)) d \tau \\
& =e^{\mathbf{L} h} u\left(t_{n}\right)+\int_{0}^{h} e^{(h-\tau) \mathbf{L}} \mathbf{N}\left(t_{n}+\tau, u\left(t_{n}+\tau\right)\right) d \tau .
\end{aligned}
$$

For approximate solution, we will use a numerical method constructed similarly to Refs. [8, 16]. Taking into account the relation $u_{j} \approx u\left(t_{j}\right)$, we define a new approximation $u_{n+1}$ of $u\left(t_{n+1}\right)$ by

$$
u_{n+1}=e^{\mathrm{L} h} u_{n}+\int_{0}^{h} e^{(h-\tau) \mathbf{L}} P_{n, k-1}\left(t_{n}+\tau\right) d \tau
$$


where $P_{n, k-1}$ is the Lagrange polynomial with the interpolation points

$$
\left(t_{n-k+1}, \mathbf{N}\left(t_{n-k+1}, u_{n-k+1}\right)\right), \cdots,\left(t_{n}, \mathbf{N}\left(t_{n}, u_{n}\right)\right) .
$$

It can be written in the form

$$
P_{n, k-1}\left(t_{n}+\theta h\right)=\sum_{j=0}^{k-1}(-1)^{j}\left(\begin{array}{c}
-\theta \\
j
\end{array}\right) \nabla^{j} \mathbf{N}_{j}, \quad \mathbf{N}_{j}=\mathbf{N}\left(t_{j}, u_{j}\right),
$$

where $\nabla$ is the backward difference operator,

$$
\nabla^{0} \mathbf{N}_{n}=\mathbf{N}_{n}, \quad \nabla^{j} \mathbf{N}_{n}=\nabla^{j-1} \mathbf{N}_{n}-\nabla^{j-1} \mathbf{N}_{n-1}, \quad j=1,2 \cdots .
$$

Substitute the polynomial (4.3) into Eq. (4.2) and obtain

$$
u_{n+1}=e^{\mathbf{L} h} u_{n}+h \sum_{j=0}^{k-1} \gamma_{j}(\mathbf{L} h) \nabla^{j} \mathbf{N}_{n}
$$

with the coefficients

$$
\gamma_{j}(z)=(-1)^{j} \int_{0}^{1} e^{(1-\theta) z}\left(\begin{array}{c}
-\theta \\
j
\end{array}\right) d \theta .
$$

The weight function (4.5) can be also represented via $\varphi$-function — viz.

$$
\varphi_{j}(z)=\frac{1}{(j-1) !} \int_{0}^{1} e^{(1-\theta) z} \theta^{j-1} d \theta, \quad j \geq 0 .
$$

Proceeding similarly to Ref. [16], we write Eq. (4.4) as

$$
u_{n+1}=e^{\mathbf{L} h} u_{n}+h \sum_{j=0}^{k} \bar{\beta}_{j} \mathbf{N}_{n-j},
$$

where the coefficients $\bar{\beta}_{j}$ are linear combinations of the weight functions $\varphi_{j}$. Note that, according to Ref. [16, Page 8], the first four coefficients for ETD Adams-Bashforth and Adams-Moulton methods are

$$
\left(\begin{array}{l}
\bar{\beta}_{1} \\
\bar{\beta}_{2} \\
\bar{\beta}_{3} \\
\bar{\beta}_{4}
\end{array}\right)=\left(\begin{array}{cccc}
1 & 11 / 6 & 2 & 1 \\
0 & -3 & -5 & -3 \\
0 & 3 / 2 & 4 & 3 \\
0 & -1 / 3 & -1 & -1
\end{array}\right)\left(\begin{array}{l}
\varphi_{1}(\mathbf{L} h) \\
\varphi_{2}(\mathbf{L} h) \\
\varphi_{3}(\mathbf{L} h) \\
\varphi_{4}(\mathbf{L} h)
\end{array}\right),
$$

and

$$
\left(\begin{array}{c}
\bar{\beta}_{0} \\
\bar{\beta}_{1} \\
\bar{\beta}_{2} \\
\bar{\beta}_{3}
\end{array}\right)=\left(\begin{array}{cccc}
0 & 1 / 3 & 1 & 1 \\
1 & 1 / 2 & -2 & -3 \\
0 & -1 & 1 & 3 \\
0 & -1 / 6 & 0 & -1
\end{array}\right)\left(\begin{array}{l}
\varphi_{1}(\mathbf{L} h) \\
\varphi_{2}(\mathbf{L} h) \\
\varphi_{3}(\mathbf{L} h) \\
\varphi_{4}(\mathbf{L} h)
\end{array}\right),
$$


respectively. The $\varphi$-functions in (4.6) can be computed by the recursive formula

$$
\varphi_{0}(z)=e^{z}, \quad \varphi_{j}(z)=\frac{\varphi_{j-1}(z)-\varphi_{j-1}(0)}{z}, \quad j \geq 1 .
$$

In order to overcome computational problems caused by formulas (4.10), we employ the Krylov projection algorithm [19]. More precisely, if $A$ is $N \times N$ matrix, then for any vector $v$ the element $\varphi(A) v$ is approximated by its projection onto Krylov subspace $K_{m}(A, v):=$ $\operatorname{span}\left\{v, A v, \cdots, A^{m-1} v\right\}$. The orthonormal basis $\left\{v_{1}, v_{2}, \cdots, v_{m}\right\}$ of $K_{m}(A, v)$ is constructed according to the modified Arnoldi iteration $[1,19]$. Hence, we have

$$
A V_{m}=V_{m} H_{m}+\bar{h}_{m+1, m} v_{m+1} e_{m}^{T},
$$

where $e_{m}=(0, \cdots, 0,1,0, \cdots, 0)^{T}$ is the unit vector with 1 as the $m$-th coordinate, $V_{m}=$ $\left[v_{1} v_{2} \cdots v_{m}\right] \in \mathbb{R}^{N \times m}$ and $\bar{h}_{m+1, m}$ is an entry of the upper Hessenberg matrix $H_{m}$,

$$
H_{m}=V_{m}^{T} A V_{m}
$$

As the result, the element $\varphi(A) v$ is approximated as

$$
\varphi(A) v \approx V_{m} V_{m}^{T} \varphi(A) V_{m} V_{m}^{T} v,
$$

so that the relations $v_{1}=v /\|v\|_{2}$ and (4.12) yield

$$
\varphi(A) v \approx\|v\|_{2} V_{m} \varphi\left(H_{m}\right) e_{1} .
$$

Now the computations can be performed with the matrices of order $m$ significantly lower than the initial order $N$.

\section{Numerical Results and Discussion}

In this section, we discuss the efficiency and accuracy of the barycentric Legendre spectral collocation (SC) method on the example of Vasicek [24] and CIR [7] models. Analytic solutions of these problems are not known, so an approximate solution computed on the grid with $N=500$ points, serves as the benchmark solution $V_{\text {benchmark }}$. In order to guaranty the space discretisation dominance in the error, the penalty parameters are set to $\epsilon=10^{-4}$, $p=3$ and the time step is $h=10^{-4}$. The relative error of the method is evaluated in $L_{2}$ and uniform norms - i.e.

and

$$
R . L_{2}:=\sqrt{\frac{\sum_{j=1}^{n}\left(V\left(r_{j}\right)-V_{\text {benchmark }}\left(r_{j}\right)\right)^{2}}{\sum_{j=1}^{n}\left(V_{\text {benchmark }}\left(r_{j}\right)\right)^{2}}},
$$

$$
\text { R.Error }:=\frac{\left|V\left(r_{j}\right)-V_{\text {benchmark }}\left(r_{j}\right)\right|}{\left|V_{\text {benchmark }}\left(r_{j}\right)\right|} \text {, }
$$


Table 1: American put option on zero-coupon bond.

\begin{tabular}{|c|c|c|c|c|c|c|c|c|}
\hline \multirow[t]{2}{*}{$\bar{N}$} & \multicolumn{4}{|c|}{ Vasicek Model } & \multicolumn{4}{|l|}{ CIR Model } \\
\hline & Price & R.Error & $R . L_{2}$ & $\mathrm{CPU}$ & Price & R.Error & R. $L_{2}$ & $\mathrm{CPU}$ \\
\hline 20 & 4.80290303 & $1.6463(-2)$ & $3.0396(-1)$ & 0.085 & 1.71387480 & $2.0038(-2)$ & $3.1138(-1)$ & 0.086 \\
\hline 40 & 4.88861445 & $1.0885(-3)$ & $4.3153(-2)$ & 0.141 & 1.74690615 & $1.1516(-3)$ & $4.3216(-2)$ & 0.142 \\
\hline 80 & 4.88321504 & $1.7230(-5)$ & $4.4141(-4)$ & 0.282 & 1.74885763 & $3.5763(-5)$ & $8.5012(-4)$ & 0.283 \\
\hline 160 & 4.88329981 & $1.2948(-7)$ & $1.3228(-5)$ & 0.817 & 1.74892032 & $8.0918(-8)$ & $3.4340(-6)$ & 0.818 \\
\hline & \multicolumn{4}{|c|}{ Benchmark price: 4.88329918} & \multicolumn{4}{|c|}{ Benchmark price: 1.74892018} \\
\hline
\end{tabular}
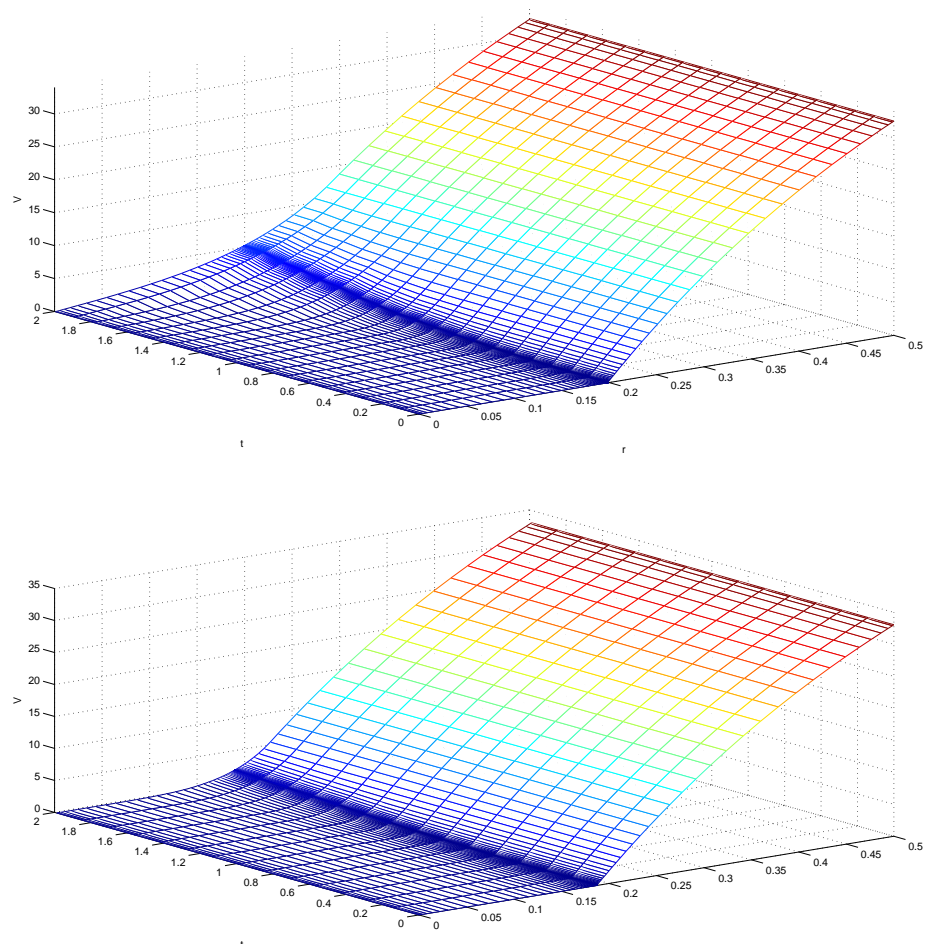

Figure 1: American put option on zero-coupon bond, $N=80, T=2$. Top: Vasicek model. Bottom: CIR model.

where $V\left(r_{j}\right)$ and $V_{\text {benchmark }}\left(r_{j}\right)$ are the computed and benchmark solutions at a point $r_{j}$.

The method has been tested for various sets of parameters. Thus we set $\kappa=0.1, \theta=$ $0.08, \sigma=0.1, E=100, K=60, T=0.5, T^{*}=5$ and restrict $r$ to the computational domain $\bar{I}=[0,0.5]$. The grid stretching parameter $\alpha$ is $10^{4}$ and "R.Error" is determined at the singularity point $K=B\left(r, T^{*}-T\right)$, i.e. for $r=-\log \left(K / A\left(T^{*}-T\right)\right) / D\left(T^{*}-T\right)$. Fig. 1 shows the value of American put options on a zero coupon-bond. The approximate solutions are quantitatively excellent. The plot show that our numerical scheme is stable.

Table 1 contains the option price, $L_{2}$ and uniform relative errors, and CPU time for the ETD method combined with spectral collocation method for Vasicek and CIR models. Computations performed on a dual-core 64-bit computer in MATLAB environment, demonstrate 

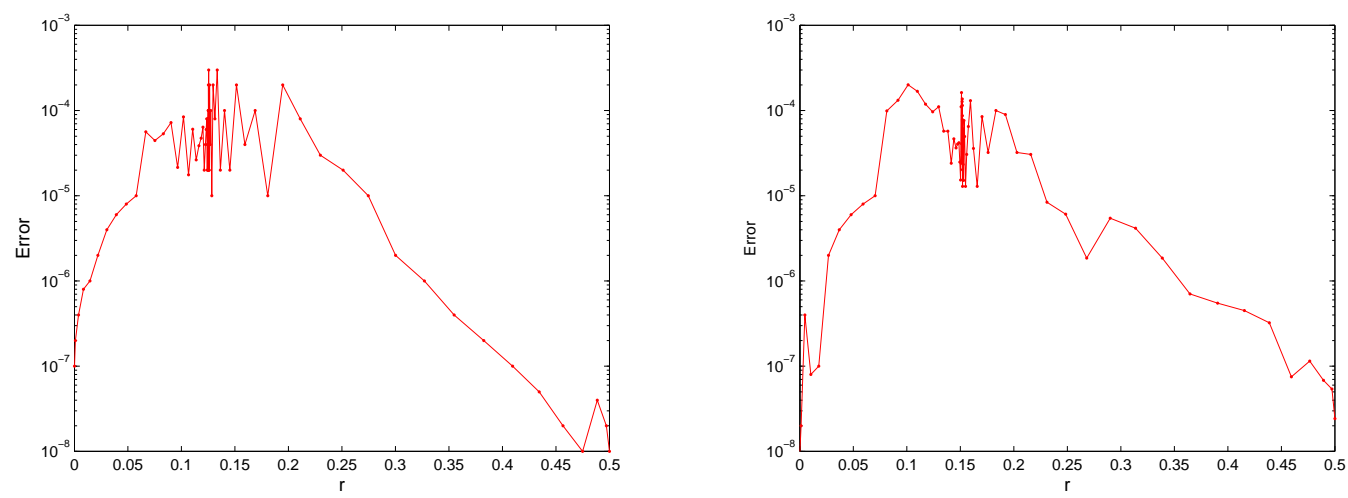

Figure 2: Absolute errors of the American put option on zero-coupon bond, $N=80, T=0.5$. Left: Vasicek model. Right: CIR model.
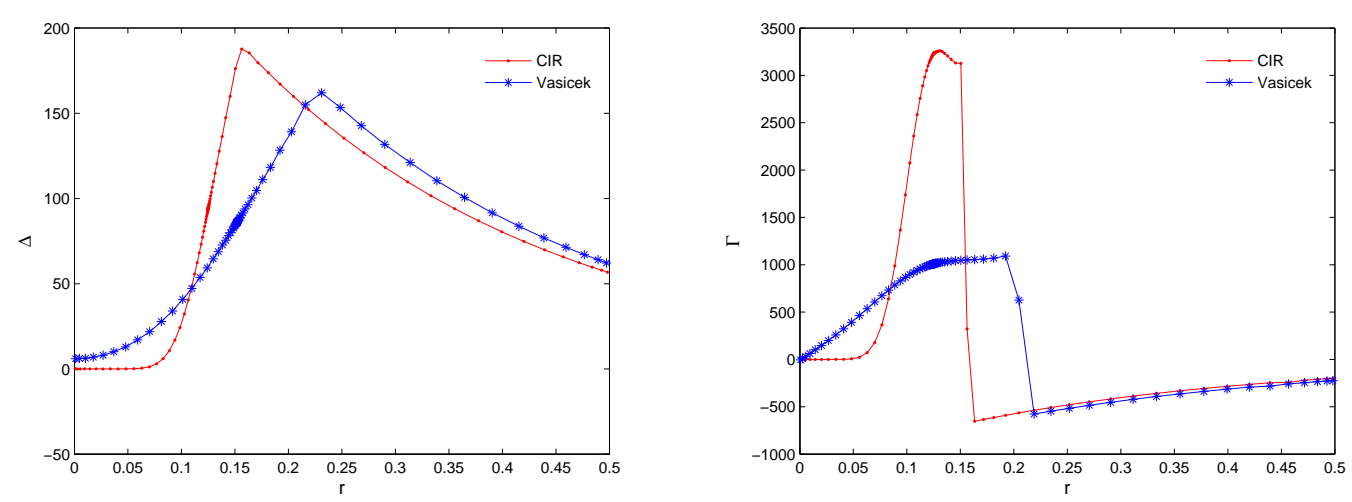

Figure 3: Value of $\Delta$ (left) and $\Gamma$ (right) of the American put option on zero-coupon bond for Vasicek (red) and CIR (blue) models, $N=80, T=0.5$.

the exponential convergence of the spectral collocation scheme. The CPU time, measured in seconds, indicates the efficiency of both models. Fig. 2 shows absolute error of the American put option on a zero-coupon bond. Fig 3 displays the Greeks ( $\Delta$ and $\Gamma$ ) of American put option on a zero-coupon bond. They measure the sensitivity of the option value with respect to the variations in the asset price and parameters associated with a model. In fact, $\Delta$ and $\Gamma$ show the changes of the option value $V$ and, correspondingly, $\Delta$ with respect to the asset price. We find these results fairly convincing. In contrast to the Crank-Nicolson method, Fig. 3 does not show any oscillation so that the above approach is robust.

\section{Concluding Remarks}

We applied the barycentric Legendre spectral collocation method to Vasicek and CIR models of American put options on a zero-coupon bond. The original problem is approximated by a nonlinear PDE with the subsequent use of an ETD method relaying on barycen- 
tric Legendre interpolation and a Krylov subspace. Numerical examples shows the stability and a good accuracy of the method.

\section{Acknowledgments}

The authors would like to thank the anonyms referees for their valuable comments and suggestions. E.P. acknowledges financial support provided by the Office of Research and Development of the University of the Western Cape. KCP was supported by the South African National Research Foundation.

\section{References}

[1] W. Arnoldi, The principle of minimized iteration in the solution of the matrix eigenvalue problem, Quart. Appl. Math. 9, 17-29 (1951).

[2] A. Bensoussan and J.L. Lions, Applications of Variationel Inequalities in Stochastic Control, North-Holland (1978).

[3] J.P. Berrut and L.N. Trefethen, Barycentric Lagrange interpolation, SIAM Rev. 46, 501-517 (2004).

[4] F. Black and P. Karashinki, Bond and option pricing when short rates are lognormal, Fin. Anal. J. 47, 277-298 (1991).

[5] M.A. Botchev, A short guide to exponential Krylov subspace time integration for Maxwell's equations, Memorandum 1992, Department of Applied Mathematics, University of Twente (2012).

[6] M.J. Brennan and E.S. Schwartz, The valuation of American put options, J. Finance 32, 449-462 (1977).

[7] J.C. Cox, J.E. Ingersoll and S.A. Ross, A theory of the term structure of interest rates, Econometrica 53, 385-407 (1985).

[8] S.M. Cox and P.C. Matthews, Exponential time differencing for stiff systems, J. Comput. Phys. 176, 430-455 (2002).

[9] M.A. Gondal, Exponential Rosenbrock integrators for option pricing, J. Comput. Appl. Math. 234, 1153-1160 (2010).

[10] P. Henrici, Essentials of Numerical Analysis with Pocket Calculator Demonstrations. Wiley (1982).

[11] M. Hochbruck and A. Ostermann, Explicit exponential Runge-Kutta methods for semi-linear parabolic problems, SIAM J. Numer. Anal. 43, 1069-1090 (2005).

[12] F. Jamshidian, An exact bond options formula, J. Finance 44, 205-209 (1989).

[13] A.K. Kassam and L.N. Trefethen, Fourth-order time stepping for stiff PDEs, SIAM J. Sci. Comput. 26, 1214-1233 (2005).

[14] P. Kovalov, V. Linetsky and M. Marcozzi, Pricing multi-asset American options: A finite element method-of-lines with smooth penalty, J. Sci. Comput. 33, 209-237 (2007).

[15] J.L. Lagrange, Leçons élémentaires sur les mathématiques, données à l'Ecole Normale en 1795, J. de l'École Polytec. 7, 183-287 (1795).

[16] B.V. Minchev, Exponential Integrators for Semilinear Problems, PhD thesis, University of Bergen (2004).

[17] N. Rambeerich, D.Y. Tangman, A. Gopaul and M. Bhuruth, Exponential time integration for fast finite element solutions of some financial engineering problems, J. Comput. Appl. Math. 224, 668-678 (2009). 
[18] R. Rebonato, Interest-rate Option Models: Understanding, Analyzing and using Models for Exotic Interest-rate Options, Wiley (1996).

[19] Y. Saad, Iterative Methods for Sparse Linear Systems, PWS Publishing Company (1996).

[20] L. ShuJin and L. ShengHong, Pricing American interest rate option on zero-coupon bond numerically, Appl. Math. Comput. 175, 834-850 (2006).

[21] T. Schmelzer and L.N. Trefethen, Evaluating matrix functions for exponential integrators via carathéodory-fejér approximation and contour integrals, Electron. Trans. Numer. Anal. 29, 118 (2007).

[22] D.Y. Tangman, A. Gopaul and M. Bhuruth, Exponential time integration and Chebychev discretisation schemes for fast pricing of options, Appl. Numer. Math. 58, 1309-1319 (2008).

[23] T.W. Tee and L.N. Trefethen, A rational spectral collocation method with adaptively transformed Chebyshev grids points, J. Sci. Comput. 28, 1798-1811 (2006).

[24] O. Vasicek, An equilibrium characterization of the term structure, J. Fin. Econom. 5, 177-188 (1977).

[25] K.R. Vetzal, Stochastic volatility, movements in short term interest rates, and bond option values, J. Bank. Finance 21, 169-196 (1997).

[26] W. Werner, Polynomial interpolation: Lagrange versus Newton, Math. Comp. 43, 205-217 (1984).

[27] H. Wang and D. Huybrechs, Explicit barycentric weights for polynomial interpolation in the roots of extrema of classical orthogonal polynomials, Report TW604, Department of Computer Science, KU Leuven (2012).

[28] P. Wilmott, Quant. Finance, Wiley (2007). 